\title{
Clinical outcomes of early gastric cardiac cancer treated with endoscopic submucosal dissection in patients with different indications
}

\section{Ting Fan}

Nanjing Drum tower Hospital Clinical College of Nanjing Medical University

\section{Qi Sun}

Nanjing Drum Tower Hosptial, The afflilated Hospital of Nanjing University Medical School

Shouli Cao

Nanjing Drum Tower Hospital, The Affiliated Hospital of Nanjing University Medical School

\section{Xiangshan Fan}

Nanjing Drum Tower Hospital, The Affiliated Hospital of Nanjing University Medical School

\section{Qin Huang}

Nanjing Drum Tower Hospital, The Affiliated Hospital of Nanjing University Medical School

\section{Shu Zhang}

Nanjing Drum Tower Hospital, The Affiliated Hospital of Nanjing University Medical School

\section{Ying Lv}

Nanjing Drum Tower Hospital, The Affiliated Hospital of Nanjing University Medical School

\section{Xiaoqi Zhang}

Nanjing Drum Tower Hospital, The Affiliated Hospital of Nanjing University Medical School

\section{Tingsheng Ling}

Nanjing Drum Tower Hospital, The Affiliated Hospital of Nanjing University Medical School

\section{Lei Wang}

Nanjing Drum Tower Hospital, The Affiliated Hospital of Nanjing University Medical School

Guifang Xu ( $\nabla$ xuguifang@njglyy.com )

Nanjing Drum Tower Hospital, The Affiliated Hospital of Nanjing University Medical School https://orcid.org/0000-0003-3963-8557

\section{Xiaoping Zou}

Nanjing Drum Tower Hospital, The Affiliated Hospital of Nanjing University Medical School

\section{Research article}

Keywords: early gastric cardiac cancer, endoscopic submucosal dissection, treatment outcome, beyond the expanded indication 
Posted Date: April 30th, 2020

DOl: https://doi.org/10.21203/rs.3.rs-18393/v1

License: (c) (i) This work is licensed under a Creative Commons Attribution 4.0 International License. Read Full License

Version of Record: A version of this preprint was published on March 12th, 2021. See the published version at https://doi.org/10.1186/s12876-021-01700-0. 


\section{Abstract}

Background: Endoscopic submucosal dissection (ESD) has been accepted as a standard treatment for early gastric cardiac cancer (EGCC). Here, we investigate the clinical outcomes of the EGCC patients who underwent ESD in different indications.

Methods: From January 2011 to October 2019, we enrolled 502 EGCC lesions from 495 patients which were resected by ESD at our center. We retrospectively analyzed the short-term and long-term clinical outcomes among different indication groups.

Results: The number of the patients in the absolute indication (Al), expanded indication (EI) and beyond the expanded indication (BEI) groups was 265, 137 and 93, respectively. The en bloc resection rate was $100 \%, 100 \%$ and $98.9 \%(P=0.185)$. The complete resection rate was $99.3 \%, 98.5 \%$ and $74.5 \%$, respectively $(P<0.001)$. During a median follow-up of 48.1 months, the lymph node metastasis rate was $0 \%, 0 \%$ and $2.3 \%(P<0.001)$. The distant metastasis rate was $0.4 \%, 0 \%$ and $2.3 \%$ ( $P=0.150)$. The fiveyear disease-specific survival rate in the BEI group was $96.6 \%$ ( $P=0.016)$, compared to $99.6 \%$ in the Al group and $100 \%$ in the El group.

Conclusion: The efficacy for ESD patients in El group was almost equal to the Al group. Patients in the BEl group showed generally favorable clinical outcomes and needed to be carefully checked after ESD. ESD may be an optional treatment for patients unsuitable for gastrectomy.

\section{Background}

Gastric cardiac cancer locates in the gastric cardia beneath the gastroesophageal junction[1]. It may cross the gastroesophageal junction to invade the distal esophagus. Our previous study showed $23.6 \%$ of early gastric cancer (EGC) was gastric cardiac carcinoma at our center, which is much higher compared to $7.0 \%$ in Japan[2] and $11.9 \%$ in western cohort[3]. Gastric cancer is the second most common cause of cancer-related death worldwide[4]. The five-year survival rate was less than $10 \%$ in the advanced stage[5]. As a result, it is urgent to perform early detection of gastric cancer through early screening[6]. Endoscopic resection should be applied for EGC[7]. Endoscopic mucosal resection (EMR) and endoscopic submucosal dissection (ESD) exhibited terrific clinical effects in the aspects of complete resection rate and survival outcomes if only the EGC lesions fit the standard and expanded indications[8]. Only a few studies have been published on the exact prevalence of early gastric cardiac cancer (EGCC) due to the rarity and a lack of widely accepted definition for gastric cardiac caner. There are some studies indicating that EGCC has the lower risk of lymph node metastasis than non-EGCC $[9,10]$, which is why endoscopic resection plays a more and more important role in EGCC treatment. It is widely accepted the EGCC patients of the Al and El group can obtain excellent treatment outcome. However, a part of specimen is confirmed with lymphovascular invasion, massive submucosal invasion, undifferentiated histology or ulceration after ESD, which lead to "beyond the expanded indication" (BEI). 
Until now, the long-term clinical consequence of the BEI group in EGCC patients has not been confirmed. We hope to evaluate the short-term and long-term treatment outcome in all EGCC patients, including the clinical application of the BEl patients.

\section{Methods}

\section{Patients}

From January 2011 to October 2019, 570 patients who underwent endoscopic resection for EGCC at the Affiliated Nanjing Drum Tower Hospital of Nanjing University Medical School were retrospectively enrolled consecutively. EGCC is defined as early cancers with epicenters located in a narrow region of about $3 \mathrm{~cm}$ below the esophagogastric junction (EGJ)[11]. EGJ is defined as the distally esophageal palisading vessels. Even if the EGJ was involved, it would be staged using the stomach cancer TNM and stage groups[12]. The exclusion criteria for entry into this study were: (1) low grade epithelial neoplasia $(n=25)$; (2) other type of carcinoma ( $n=10)$; (3) no carcinoma $(n=28)$; (4) multiple lesions in different indications $(n=4)$; $(5)$ insufficient data $(n=8) .495$ patients with 502 lesions were enrolled at last. A detailed flow chart on patient selection was exhibited in Supplementary Figure 1.

\section{Endoscopic resection}

Each patient underwent a routine gastroscopy to evaluate the status of margin and invasion depth of the EGCC lesion. Abdominal enhanced CT and endoscopic ultrasonography (EUS) were used to determine pretreatment tumor staging. All patients were intravenously anesthetized with midazolam and propofol before endoscopic resection. The ESD procedure was performed by 5 experienced endoscopists at our center with a standard procedure. The standard procedure is as follows: demarcation by indigo-carmine chromoendoscopy and NBI magnifying endoscopy; marking around the lesion; a circular mucosal incision around the marking spots and submucosal resection using a Dual knife step by step. Freshly endoscopically excised specimens were nailed on a dental wax plate, pictured, routinely measured, fixed overnight in $10 \%$ neutral formalin buffer then. Next, the specimens were cut evenly into 2 -mm intervals. Size of the lesions, depth of tumor invasion, tumor differentiation, lymphovascular invasion and ESD resection margin were examined (Supplementary Figure 2).

\section{Definition}

EGCC patients were divided into absolute, expanded, beyond the expanded indication group according to the classification of the Japanese Gastric Cancer Association[8]. Absolute indication was defined as differentiated mucosal cancer smaller than $2 \mathrm{~cm}$ in diameter without ulcer. Expanded indications cover one of the circumstances: 1). Differentiated mucosal adenocarcinoma larger than $2 \mathrm{~cm}$ without ulceration or lymphovascular invasion; 2). Differentiated ulcer-positive mucosal cancer smaller than $3 \mathrm{~cm}$ in size without lymphovascular invasion; 3). Poorly differentiated or undifferentiated ulcer-negative 
mucosal tumor less than $2 \mathrm{~cm}$ in size without lymphovascular invasion; 4). Differentiated ulcer-negative cancer with submucosal invasion less than $500 \mu \mathrm{m}$ in depth smaller than $3 \mathrm{~cm}$ without lymphovascular invasion. When it does not meet absolute and expand criteria, it is considered to be beyond the expanded indication[7].

En bloc resection referred to continuous monolithic resection after endoscopic resection. Complete resection was defined as endoscopic en bloc resection without histopathological evidence of tumor involvement of margins. Curative resection referred to en bloc resection, tumor size less than $2 \mathrm{~cm}$, histologically of differentiated type, pT1a, no residual tumor at the margin and no lymphovascular invasion. For lesions under expanded indication, the resection is considered as curative when all of the following condition are fulfilled: en bloc resection, negative horizontal and vertical margin and no lymphovascular infiltration[7].

The ESD-related complications included significant bleeding, perforation, and stenosis. Significant bleeding was a drop of over $2 \mathrm{~g} / \mathrm{dL}$ hemoglobin or clinical features instance of endoscopic visible melena or hematemesis. According to whether significant bleeding occurred over 48 hours after ESD, we divided it into early and late delayed bleeding[13]. When we observed a hole in the stomach wall under endoscope or a chest X-ray that found free air of the abdomen, it could be diagnosed as a perforation. When the endoscope couldn't reach the cardiac through distal esophagus because of the excessive contraction of the cardiac, we considered that a stenosis had occurred[14].

Local recurrence was diagnosed once a new tumor was discovered at the prior ESD site. Tumors that were detected at other sites within 1 year after the ESD were interpreted as synchronous tumors, while tumors that were revealed at other sites more than 1 year after the ESD procedure were termed metachronous tumors.

\section{eCura system}

We evaluated the lymph node metastasis rate of EGCC patients after ESD by eCura system. The eCura system consists of five clinicopathological factors, which are scored as followed: 1 point each for tumor size $>30 \mathrm{~mm}$, positive vertical margin, venous invasion, and submucosal invasion over $500 \mu \mathrm{m}$ and three points for lymphatic invasion. And the patients are divided into three groups according the total points: low risk (0-1 points), intermediate risk (2-4 points) and high risk (5-7 points)[15]. In this study, we used the eCura system to access the disease specific survival and cancer recurrence rate between the two BEI patients with or without additional surgery.

\section{Follow-up}

Patients were required scheduled endoscopy examination in the first 3, 6, 12 months after ESD procedure, and once a year thereafter. The surveillance of thoracic and abdominal CT was performed after 6,12 
months. When endoscopists found a new tumor at a previous ESD location, local recurrence was considered to occur. Synchronized tumors were diagnosed once tumors were found at other sites within a year of ESD, and metachronous tumors were that occurred at the other sites over a year after ESD.

\section{Statistical analysis}

Statistics was analyzed by SPSS 23.0 (IBM, Armonk, New York, USA). Student's tor Chi-square test was applied to assess the differences between measurement data. Chi-square test or Fisher's exact test was called for the analyses of categorical variables to obtain statistical significance. Kaplan-Meier method was used to estimate the survival rates. A $p$ value of $<0.05$ was regarded as statistically significant.

\section{Results}

\section{Baseline Demographic and Clinical Features}

A total of 570 patients had undergone ESD and 495 patients with 502 lesions were enrolled with the following criteria, among whom 265 patients with 271 lesions were enrolled in the Al group, 137 patients with 137 lesions in the El group and 93 patients with 94 lesions in the BEl group. 5 patients were withdrawn during the follow-up. 450 patients were enrolled for the long-term outcome analysis in the end (Supplementary Figure 3).

As shown in Table 1, the average age was 65.5 (range, 44-87) and 82\% were male. 291 patients (58.8\%) had a smoking history, 263 patients (53.1\%) had a drinking habit and 230 patients (46.5\%) regularly ate pickled food. Nearly half of the patients $(40.8 \%)$ had a family tumor history. The most common complication was hypertension $(32.7 \%, 162 / 495) .14$ patients $(2.8 \%)$ suffered from gastroesophageal reflux disease.

\section{Endoscopic and Pathological Characteristics}

The average tumor size was $12 \mathrm{~mm}$ (range, 2-20) in the Al group, $23 \mathrm{~mm}$ (range, 6-66) in the El group, and $27 \mathrm{~mm}$ (range, 5-65) in the BEl group $(P<0.001)$ (Table 2). Most lesions were smaller than $20 \mathrm{~mm}$ (73.7\%, 370/502). The most common site of EGCC was posterior curvature $(51.0 \%, 256 / 502)$, followed by lesser curvature $(42.2 \%, 212 / 502)$. The most common endoscopic infiltration growth pattern was INFa $(66.3 \%, 333 / 502)$. The most common macroscopic pattern, in the descending order, was 0 -Ilc $(45.2 \%$, 227/502), 0-lla+llc (27.9\%, 140/502), 0-lla (14.9\%, 75/502), 0-Ilb (10.0\%, 50/502), 0-III (1.2\%, 6/502), and $0-1(0.8 \%, 4 / 502)$. All tumor lesions in the Al group were intramucosal and differentiated. Instead, in the BEl group, 5 patients $(5.3 \%, 5 / 94)$ had undifferentiated carcinoma and the majority $(94.6 \%, 89 / 94)$ had submucosal invasion $(P<0.001) .24$ patients $(4.8 \%, 24 / 502)$ had ulceration over the three group and 13 patients $(13.8 \%, 13 / 94)$ in the BEl group were positive. Focal distal esophageal involvement was detected in $12.0 \%(60 / 502)$ in all patients. Lymphovascular invasion was identified in $3.0 \%(15 / 502)$ in all patients. 
Gastritis cystica profunda was found in over $24 \%$ of lesions (122/502) and there were no differences among the three groups. Overall positive rate of helicobacter pylori was $56.8 \%$ and the difference among the three groups was non-significant. Atrophic gastric carditis was found in $89.4 \%$ (449/502).

\section{Short-term Outcomes and Complications based on indication}

As table 3 clarified, the overall en bloc resection rate was 99.8\% (501/502), among which only one lesion in the BEl group was broken. Complete resection rate of all cases was 94.4\% (474/502); 99.3\% (269/271), $98.5 \%$ (135/137) and 74.5\% (70/94), respectively $(P<0.001)$. The rate of curative resection was $79.9 \%$ in all the patients: $98.5 \%(267 / 271), 97.8 \%(134 / 137)$ and $0 \%(0 / 94)$, respectively $(P<0.001)$. Factors associated non-curative resection included lymphovascular infiltration $(n=15)$, submucosal invasion ( $n=$ 71), positive vertical margin $(n=4)$, positive lateral margin $(n=21)$, or positive lateral and vertical margin $(n=3)$.

In the aspect of complications, 12 cases had significant bleeding, in which 8 cases were early delayed bleeding, 4 cases were late delayed bleeding. The early delayed bleeding rate was $0.4 \%(1 / 271), 1.5 \%$ (2/137), 5.3\% (5/94) in the Al, El and BEl group, respectively, which was a difference of statistics among the three groups $(P=0.004)$. This could be due to the larger size of tumor lesions and submucosal invasion. Luckily, all the patients who suffered from bleeding were successfully managed by endoscopic hemostasis $(n=11)$ and the use of hemostatic drugs such as octreotide acetate and somatostatin ( $n=$ 1). Perforation was found in $1 \mathrm{Al}$ patient and this patient's symptoms improved after conservative treatment. Stenosis was a common complication of patients who underwent ESD and was found in $3.6 \%$ $(18 / 502)$ patients, among whom 4 patients $(1.5 \%, 4 / 271)$ were in the Al group, 10 patients $(7.3 \%, 10 / 137)$ belonged to the El group and 4 patients $(4.4 \%, 4 / 94)$ were members of the BEl group $(P=0.002)$. After balloon dilation, the symptoms of stenosis were easily improved. The presence of stenosis was positively related to a circumferential extent of the mucosal defect of $>3 / 4$ or longitudinal extent of $>5 \mathrm{~cm}$ in length according to the previous investigation[16]. The median time of ESD operations was 65.3 minutes (range 10-246), while the BEI patients required longer procedure time, which was 72.9 minutes (range 18-223) $(P=0.007)$. The average hospitalization time was 6.6 days (range $2-19)$. Similarly, the El and BEI patients stayed longer than the Al patients $(P=0.012)$.

\section{Long-term therapeutic outcomes according to the indication}

During the median follow-up of 48.1 months (range 18-101), 45 patients $(9.1 \%, 45 / 495)$ were lost during follow-up. Additional surgery was carried out for 7 Al patients $(2.9 \%, 7 / 239), 10$ El patients $(8.1 \%, 10 / 124)$ and $42 \mathrm{BEl}$ patients $(48.3 \%, 42 / 87)$ (Table. 3$)$. Local recurrence was found in only one Al patient because of the residual tumor $(P=1.000)$. Synchronous cancer was detected in 39 patients $(8.7 \%, 39 / 450)$, and 
there was no statistically significant difference among the three groups. The similar conclusion was achieved for metachronous cancer. During the follow-up, 2 BEl patients $(2.3 \%, 2 / 87)$ were found to have lymph node metastasis $(P=0.037)$. These patients underwent the additional surgery and survived well during long-term follow-up. Distant metastasis was developed in one Al patient and two BEl patients (Table.4). They didn't receive the additional surgery and passes away unfortunately. Disease-specific death occurred in 4 patients $(0.9 \%, 4 / 450) ; 1(0.4 \%, 1 / 239)$ in the Al group, no patient in the El group and $3(3.4 \%, 3 / 87)$ in the BEl group $(P=0.030)$. The Al patient was dead because of metastasis whose lateral margin was positive and he refused the additional surgery. One BEl patient was dead due to the significant bleeding after the additional surgery. The other two patients passed away related to distant metastasis without additional surgery (Table 5). Five-year overall survival rates were $96.1 \%$ (Al), $98.3 \%$ (El) and $89.1 \%(\mathrm{BEI})$, which didn't have any statistic difference $(P=0.180)$ (Fig.1 A). Five-year diseasespecific survival rates of three groups were $99.6 \%, 100 \%$ and $96.6 \%$ in the Al, El and BEl group, respectively, which was significantly different between each other $(P=0.016)$ (Fig.1 B). Besides, we analyzed whether the additional surgery was necessary for the patients in the BEl group. After ESD surgery, 93 patients were enrolled into the BEl group based on postoperative pathology. 6 patients were lost during the follow-up. 42 of the remaining $87 \mathrm{BEl}$ patients underwent additional surgery according to the doctor's advice. As we can see in Fig. $1 \mathrm{C}$ and D, the additional surgery didn't have an influence on neither the overall survival nor the disease specific survival.

We wondered why the disease specific survival was no significantly difference between the two BEI group with or without the additional surgery. So, we applied the eCura system to evaluate whether there was any difference between these two group. According to the generally accepted method, we divided the BEI patients with or without additional surgery into three categories (Supplementary Table 1). The detailed scores had no difference between the two groups (Fig.1 E), which meant the LNM risk was resemble between the BEl patients with or without surgery, consistent with the survival analysis. In low risk category, disease specific survival was higher in the patient accepted the additional surgery $(100 \%$ vs $97.1 \%)$, but was no significant difference for the two groups ( $p=0.389 ;$ Fig. $1 \mathrm{~F})$. In the intermediate risk category, we could achieve the similar conclusion: the patients received the additional surgery has higher five-year disease-free survival rate, but there was no significantly different $(92.9 \%$ vs $88.9 \%, p=0.830$;

Fig. $1 \mathrm{G}$ ). Since there was no disease-related death of the patients in the high risk category and a small number of cases, we could not achieve a reliable survival analysis for the data (Fig. $1 \mathrm{H}$ ). In summary, the patients in the BEl group may not benefit from the additional surgery after ESD in our research.

\section{Discussion}

This is a large-scale research to compare the short- and long-term clinical outcomes among the absolute indication, expanded indication and beyond the expanded indication EGCC patients of endoscopic resection. Across hospitals all over the world, endoscopic resection has gain widely acceptation as a standard treatment of EGC under absolute and expanded criteria as an alternative to surgical resection of distal esophagus and proximal stomach[17-21]. ESD has advantages in lower rates of acute complications and comparable overall survival[20]. Several studies have displayed the effectiveness and 
safety of endoscopic resection for adenocarcinoma of EGJ[22, 23]. For the cancer located in EGJ, it encompasses both gastric cardia adenocarcinoma (GEA) and Barrett's esophageal adenocarcinoma $\triangle B E A \rrbracket$ due to short-segment Barrett's esophagus. Few researches have specially focused on the clinical outcome of ESD for these gastric cardiac adenocarcinomas. Osumi et al. reported curative resection rate was higher in GCA group (81\%) than BEA group (60\%)[24]. Jang et al. enrolled 82 patients with gastric cardiac tumors and the en bloc resection, complete resection, and curative resection rates were $87 \%, 79 \%$, and $66 \%[25]$. A handful of researches have proved ESD is an efficient way to cure EGCC patients. Due to the small sample size, more persuasive studies needed to be conducted.

In our study, we enrolled 495 patients with 502 ESD-related EGCC lesions. The effectiveness of ESD was confirmed by comparing the short and long-term clinical consequence among the Al, El and BEl groups. No significant difference was found in demographic statistics for these three groups. In the present study, patients in $\mathrm{BEl}$ group had more family history than $\mathrm{Al}$ and $\mathrm{El}$ groups $(P=0.018)$. Increased tumor size, deeper tumor invasion, presence of ulcer and poor differentiated were significantly different among the three groups, which was consistent with the definition. The HP positive patients were diagnosed by (13)Curea breath test or rapid urease tests. Multiple epidemiological studies came to the similar finding that prevalence of HP infection was about $50 \%$ in developing countries[26, 27]. H. pylori may also be a cause or co-factor for gastric cancer, and eradication of $H$. pylori infection was associated with a reduced incidence of gastric cancer[28]. Therefore, we recommended the patients undergo a quadruple therapy to eradicate $H$. pylori. In our research, atrophy of the mucosa around the tumor lesion could be found in the vast majority EGCC patients. Atrophic gastritis and intestinal metaplasia were the most significant risk factors for gastric cancer[29,30], thus the endoscopic surveillance in these high risk patients were expected to be extremely important.

The complete resection and curative resection rates in the Al group were meaningfully higher in comparison with the other two groups $(P<0.001)$. This was closely related to the positive vertical and lateral cutting margins in the El and BEl groups. Suzuki et al. drew the conclusion through their research that positive margins with submucosal infiltration (odds ratio 3.6) and lymphovascular invasion (odds ratio 3.5) had significant correlation with lymph node metastasis and patients who didn't meet curative resection especially with lymphovascular invasion or positive margin with submucosal invasion should receive additional gastrectomy[31]. Positive lateral margin was related closely to a mixed-type carcinoma, larger than $3 \mathrm{~cm}$ in size and the upper one third of stomach, reported by Fu et al[32]. However, there is no research about the risk factor of vertical and lateral incomplete resection in EGCC patients which needs to study in depth.

In the Al and El group, the five-year overall and disease-specific survival rates were no statistic differences with $96.1-98.3 \%$ and $99.6-100 \%$. No case in the Al and El group was found lymph node metastasis until now. Only one Al patient died because of a positive vertical margin without additional surgery. These findings were consistent with the previous results and the five-year survival rate of ESD surgery was comparable to that of radical gastrectomy[33-35]. The five-year overall survival rate of mucosal EGC was $99 \%$ and the five-year overall survival rate of submucosal EGC was $96 \%$ by surgical treatment according 
to the National Cancer Center of Japan[7], which was consistent with the long-term outcome of EGCC patients after ESD at our center. In short, if only the tumor lesions met the absolute or expanded indication, endoscopic resection could achieve satisfactory safety and efficacy from the long-term followup. As a consequence, conclusion could be drawn that endoscopic resection was an effective way for the EGCC patients within the Al and El group similar to other studies[3, 36, 37].

However, there are still many concerns in terms of the BEl group. In this study, the rate of en bloc resection arrived at $98.9 \%$ (93/94) in BEl group. Yet, The BEl group showed lower rates of complete resection $(74.5 \%, 70 / 94)(P<0.001)$. The reason for the result was mainly related to tumor lesion size, lymphatic/vascular invasion, deep submucosal invasion and undifferentiated histology. These factors were closely related to lymph node metastasis and recurrence[38-40]. As a result, these patients were not suitable for endoscopic resection from the results of previous researches. We evaluated the tumor lesions by EUS, CT or biopsy before ESD and we found some cases were under massive submucosal invasion. Gastrectomy, especially proximal gastrectomy, is the most common surgical treatment of gastric cardiac cancer for the patients unsuitable for ESD. However, some patients suffered from severe postoperative complications, such as anastomotic leakage, reflux esophagitis and anastomotic stricture after proximal gastrectomy. According to the recent reports, the short-term and long-term gastrectomy complications rates could be up to $20 \%$ compared with less than $10 \%$ by $\operatorname{ESD}[41,42]$. These patients were aware of beyond the expanded criteria but they still insisted endoscopic resection when they met advanced age, serious underlying diseases and chose the less invasive strategy instead of the surgery. We found that the five-year overall survival rate and the disease specific survival rate for the BEl group patients up to $89.1 \%$ and $96.6 \%$ during the long-term follow-up at our center. In other centers, endoscopists found the similar results which the five-year disease-free survival rate of BEl patients after ESD could be as high as $90 \%$ or more, which was quite effective[33, 43,44$]$. The rates of $\mathrm{R} 0$ resection and curative resection in BEI group may be lower than the patients within the indications, but in the long run, the survival rate in the BEI group patients who underwent ESD instead of radical gastrectomy still showed a favorable performance. Endoscopists would advise the BEI patients to receive additional surgery after ESD. Between the two groups with or without surgery, we concluded that there was no significant statistical difference from the K-M survival curve. Different from the previous studies[41, 45], those patients under BEI group didn't benefit from the additional surgery neither the five-year overall survival nor the five-year disease-specific survival. Based on the eCura system, it could be a useful aid for selecting the appropriate treatment strategy after the noncurative ESD for EGCC[40]. If we followed up long enough or enrolled more cases, we might get a different conclusion.

Our study still has several limitations. First, this research is a single center retrospective cohort analysis which may leads to a section bias and referral bias. Besides, an average of 48.1 months of follow-up may not allow us to find the significant difference in the survival rate of the BEl patients with or without additional surgery. In this rather small subgroup, the survival did not show significant differences possibly because of the minor case number. In addition, we are urge to learn about the risk factors of positive margins and noncurative resection. 
In conclusion, endoscopic resection of EGCC could achieve a favorable short-term and long-term prognosis for patients with absolute and expanded indication. Patients in beyond the expanded indication showed generally favorable clinical outcomes and needed to be carefully checked after ESD. ESD may be an optional treatment for the patients unsuitable for gastrectomy.

\section{List Of Abbreviations}

EGC: early gastric cancer; EGCC: early gastric cardiac cancer; EGJ: esophagogastric junction; ESD: endoscopic submucosal dissection; EMR: endoscopic mucosal resection; Al: absolute indication; El: expanded indication; BEl: beyond the expanded indication.

\section{Declarations}

\section{Ethics approval and consent to participate}

The study protocol was approved by the Human Ethics Review Committees of Nanjing Drum Tower Hospital.

\section{Consent for publication}

All the authors agreed to publish the research article.

\section{Availability of data and materials}

The datasets used during the current study are available from the corresponding author on reasonable request.

\section{Competing interests}

None declared.

\section{Funding}

This work was supported by grants from the National Natural Science Foundation of China (Grant Nos. 81572338 and 81672380, 81201909, 81602089), the Nanjing Medical Science and Technology Development Program (Nos. YKK 12072, YKK 15061 and YKK 16078). This work was also supported by the C-class sponsored research project of the Jiangsu Provincial Six talented Peaks (WSN-078). The funders had a role in study design, decision to publish and preparation of the manuscript.

\section{Authors' contributions}

$\mathrm{XZ}$ and $\mathrm{GX}$ designed the research; $\mathrm{XZ}, \mathrm{YL}, \mathrm{LW}$ and TL performed surgeries; $\mathrm{QS}, \mathrm{QH}$ and $\mathrm{XF}$ analyzed the pathological section; $S C$ and $S Z$ interpreted the results; TF drafted manuscript. TF, QS, SC, XF, QH, SZ, YL, $X Z, T L, L W, G X$ and $X Z$ approved final version of manuscript. 


\section{Acknowledgement}

Thanks to all the people participated in this article and the support of the hospital and department.

\section{References}

1. Huang Q, Fang C, Shi J, et al. Differences in Clinicopathology of Early Gastric Carcinoma between Proximal and Distal Location in 438 Chinese Patients. Sci. Rep. 2015;5:13439.

2. Hoteya S, Matsui A, lizuka T, et al. Comparison of the clinicopathological characteristics and results of endoscopic submucosal dissection for esophagogastric junction and non-junctional cancers. Digestion 2013;87:29-33.

3. Tate DJ, Klein A, Sidhu M, et al. Endoscopic submucosal dissection for suspected early gastric cancer: absolute versus expanded criteria in a large Western cohort (with video). Gastrointest. Endosc. 2019;90:467-479.e4.

4. Siegel RL, Miller KD, Jemal A. Cancer statistics, 2018. CA Cancer J. Clin. 2018;68:7-30.

5. Arnold M, Moore SP, Hassler S, et al. The burden of stomach cancer in indigenous populations: a systematic review and global assessment. Gut 2014;63:64-71.

6. Li S, Chung DC, Mullen JT. Screening high-risk populations for esophageal and gastric cancer. J. Surg. Oncol. 2019;120:831-846.

7. Japanese Gastric Cancer Association. Japanese gastric cancer treatment guidelines 2014 (ver. 4). Gastric Cancer 2017;20:1-19.

8. Japanese classification of gastric carcinoma: 3rd English edition. Gastric Cancer 2011;14:101-12.

9. Kim SM, Lee H, Min BH, et al. A prediction model for lymph node metastasis in early-stage gastric cancer: Toward tailored lymphadenectomy. J. Surg. Oncol. 2019;120:670-675.

10. Fang C, Shi J, Sun Q, et al. Risk factors of lymph node metastasis in early gastric carcinomas diagnosed by WHO criteria in 379 Chinese patients. J. Dig. Dis. 2016;17:526-537.

11. Q. Huang, Y. Cheng, L. Chen, et al. Low risk of lymph node metastasis in 495 early gastric cardiac carcinomas: a multicenter clinicopathologic study of 2101 radical gastrectomies for early gastric carcinoma. Modern pathology : an official journal of the United States and Canadian Academy of Pathology, Inc 2018;31:1599-1607.

12. T. W. Rice, H. Ishwaran, M. K. Ferguson, et al. Cancer of the Esophagus and Esophagogastric Junction: An Eighth Edition Staging Primer. Journal of thoracic oncology : official publication of the International Association for the Study of Lung Cancer 2017;12:36-42.

13. Azumi M, Takeuchi M, Koseki Y, et al. The search, coagulation, and clipping (SCC) method prevents delayed bleeding after gastric endoscopic submucosal dissection. Gastric Cancer 2019;22:567-575.

14. Choi JY, Park YS, Na G, et al. Safety and effectiveness of endoscopic mucosal resection or endoscopic submucosal dissection for gastric neoplasia within 2 days' hospital stay. Medicine 2019;98:e16578. 
15. Hatta W, Gotoda T, Oyama T, et al. A Scoring System to Stratify Curability after Endoscopic Submucosal Dissection for Early Gastric Cancer: "eCura system". The American journal of gastroenterology 2017;112:874-881.

16. Coda S, Oda I, Gotoda T, et al. Risk factors for cardiac and pyloric stenosis after endoscopic submucosal dissection, and efficacy of endoscopic balloon dilation treatment. Endoscopy 2009;41:421-6.

17. Ahn JY, Jung HY, Choi KD, et al. Endoscopic and oncologic outcomes after endoscopic resection for early gastric cancer: 1370 cases of absolute and extended indications. Gastrointest. Endosc. 2011;74:485-93.

18. Toyonaga T, Man-i M, East JE, et al. 1,635 Endoscopic submucosal dissection cases in the esophagus, stomach, and colorectum: complication rates and long-term outcomes. Surg. Endosc. 2013;27:1000-8.

19. Oda I, Saito D, Tada M, et al. A multicenter retrospective study of endoscopic resection for early gastric cancer. Gastric Cancer 2006;9:262-70.

20. Hahn KY, Park CH, Lee YK, et al. Comparative study between endoscopic submucosal dissection and surgery in patients with early gastric cancer. Surg. Endosc. 2018;32:73-86.

21. Pyo JH, Lee H, Min BH, et al. Long-Term Outcome of Endoscopic Resection vs. Surgery for Early Gastric Cancer: A Non-inferiority-Matched Cohort Study. The American journal of gastroenterology 2016;111:240-9.

22. Gong EJ, Kim DH, Ahn JY, et al. Comparison of long-term outcomes of endoscopic submucosal dissection and surgery for esophagogastric junction adenocarcinoma. Gastric Cancer 2017;20:84-91.

23. C. H. Park, E. H. Kim, H. Y. Kim, et al. Clinical outcomes of endoscopic submucosal dissection for early stage esophagogastric junction cancer: a systematic review and meta-analysis. Digestive and liver disease : official journal of the Italian Society of Gastroenterology and the Italian Association for the Study of the Liver 2015;47:37-44.

24. Osumi H, Fujisaki J, Omae M, et al. Clinicopathological features of Siewert type II adenocarcinoma: comparison of gastric cardia adenocarcinoma and Barrett's esophageal adenocarcinoma following endoscopic submucosal dissection. Gastric Cancer 2017;20:663-670.

25. Jang YS, Lee BE, Kim GH, et al. Factors Associated With Outcomes in Endoscopic Submucosal Dissection of Gastric Cardia Tumors: A Retrospective Observational Study. Medicine 2015;94:e1201.

26. Pan KF, Zhang L, Gerhard M, et al. A large randomised controlled intervention trial to prevent gastric cancer by eradication of Helicobacter pylori in Linqu County, China: baseline results and factors affecting the eradication. Gut 2016;65:9-18.

27. Zamani M, Ebrahimtabar F, Zamani V, et al. Systematic review with meta-analysis: the worldwide prevalence of Helicobacter pylori infection. Aliment. Pharmacol. Ther. 2018;47:868-876.

28. Lee YC, Chiang TH, Chou CK, et al. Association Between Helicobacter pylori Eradication and Gastric Cancer Incidence: A Systematic Review and Meta-analysis. Gastroenterology 2016;150:11131124.e5. 
29. Park YH, Kim N. Review of atrophic gastritis and intestinal metaplasia as a premalignant lesion of gastric cancer. Journal of cancer prevention 2015;20:25-40.

30. Yoon H, Kim N. Diagnosis and management of high risk group for gastric cancer. Gut and liver 2015;9:5-17.

31. Haruhisa Suzuki, Ichiro Oda, Seiichiro Abe, et al. Clinical outcomes of early gastric cancer patients after noncurative endoscopic submucosal dissection in a large consecutive patient series. Gastric Cancer 2017;20:679-689.

32. Fu QY, Cui Y, Li XB, et al. Relevant risk factors for positive lateral margin after en bloc endoscopic submucosal dissection for early gastric adenocarcinoma. J. Dig. Dis. 2016;17:244-51.

33. Sohn SH, Lee SH, Kim KO, et al. Therapeutic outcomes of endoscopic submucosal dissection for early gastric cancer: single-center study. Eur. J. Gastroenterol. Hepatol. 2017;29:61-67.

34. Chung IK, Lee JH, Lee SH, et al. Therapeutic outcomes in 1000 cases of endoscopic submucosal dissection for early gastric neoplasms: Korean ESD Study Group multicenter study. Gastrointest. Endosc. 2009;69:1228-35.

35. Isomoto H, Shikuwa S, Yamaguchi N, et al. Endoscopic submucosal dissection for early gastric cancer: a large-scale feasibility study. Gut 2009;58:331-6.

36. A. Probst, A. Schneider, T. Schaller, et al. Endoscopic submucosal dissection for early gastric cancer: are expanded resection criteria safe for Western patients? Endoscopy 2017;49:855-865.

37. Kim SG, Park CM, Lee NR, et al. Long-Term Clinical Outcomes of Endoscopic Submucosal Dissection in Patients with Early Gastric Cancer: A Prospective Multicenter Cohort Study. Gut and liver 2018;12:402-410.

38. Li H, Huo ZB, Kong FT, et al. Predictive factors for lymph node metastasis and defining a subgroup treatable for laparoscopic lymph node dissection after endoscopic submucosal dissection in poorly differentiated early gastric cancer. World. J. Gastrointest. Oncol. 2018;10:360-366.

39. Abdelfatah MM, Barakat $\mathrm{M}$, Lee $\mathrm{H}$, et al. The incidence of lymph node metastasis in early gastric cancer according to the expanded criteria in comparison with the absolute criteria of the Japanese Gastric Cancer Association: a systematic review of the literature and meta-analysis. Gastrointest. Endosc. 2018;87:338-347.

40. Niwa H, Ozawa R, Kurahashi $Y$, et al. The eCura system as a novel indicator for the necessity of salvage surgery after non-curative ESD for gastric cancer: A case-control study. PLoS One 2018;13:e0204039.

41. Fukunaga S, Nagami Y, Shiba M, et al. Long-term prognosis of expanded-indication differentiatedtype early gastric cancer treated with endoscopic submucosal dissection or surgery using propensity score analysis. Gastrointest. Endosc. 2017;85:143-152.

42. Ahn SH, Jung DH, Son SY, et al. Laparoscopic double-tract proximal gastrectomy for proximal early gastric cancer. Gastric Cancer 2014;17:562-70.

43. Kakushima N, Hagiwara T, Tanaka M, et al. Endoscopic submucosal dissection for early gastric cancer in cases preoperatively contraindicated for endoscopic treatment. United European 
gastroenterology journal 2013;1:453-60.

44. Suzuki H, Oda I, Nonaka S, et al. Is endoscopic submucosal dissection an effective treatment for operable patients with clinical submucosal invasive early gastric cancer? Endoscopy 2013;45:93-7.

45. Jeon MY, Park JC, Hahn KY, et al. Long-term outcomes after noncurative endoscopic resection of early gastric cancer: the optimal time for additional endoscopic treatment. Gastrointest. Endosc. 2018;87:1003-1013.e2.

\section{Tables}

Due to technical limitations, Tables 1-5 are provided in the Supplementary Files section.

\section{Figures}


A

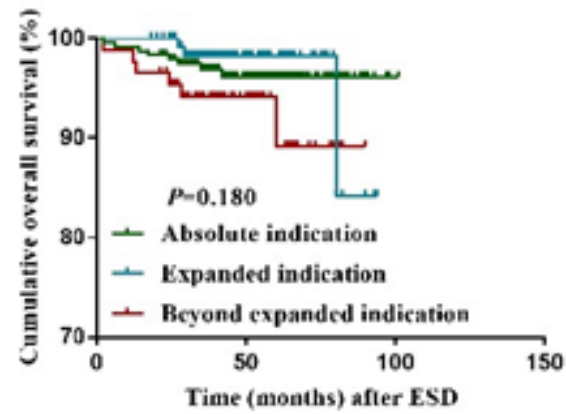

$\mathrm{C}$

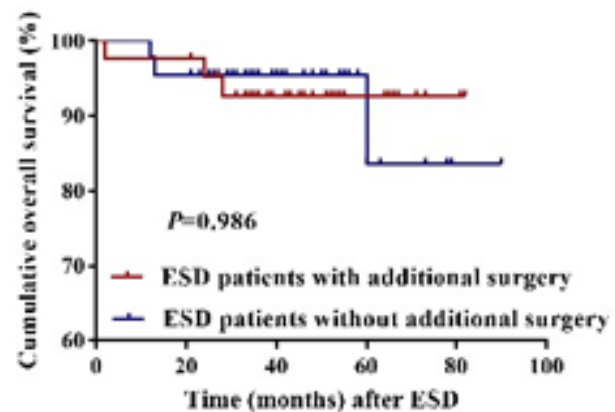

$\mathrm{E}$

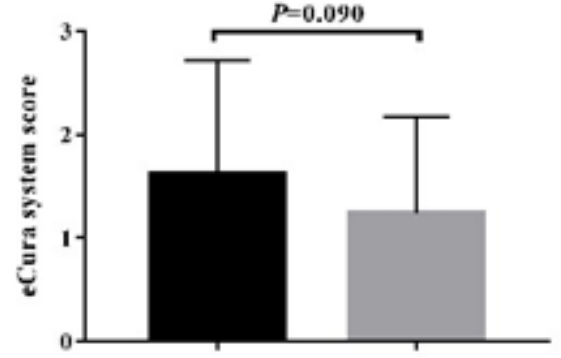

G

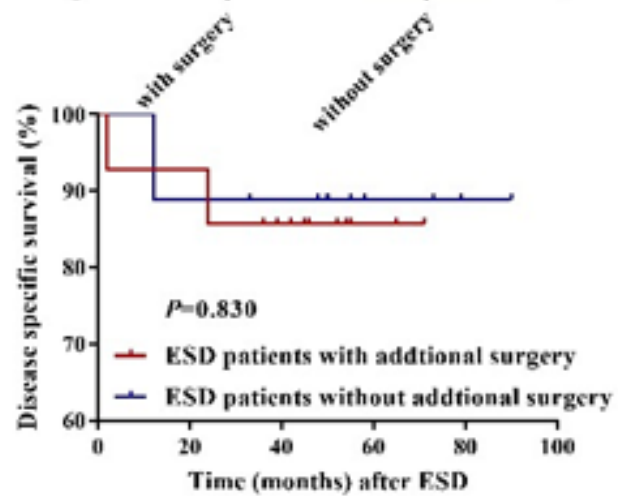

B

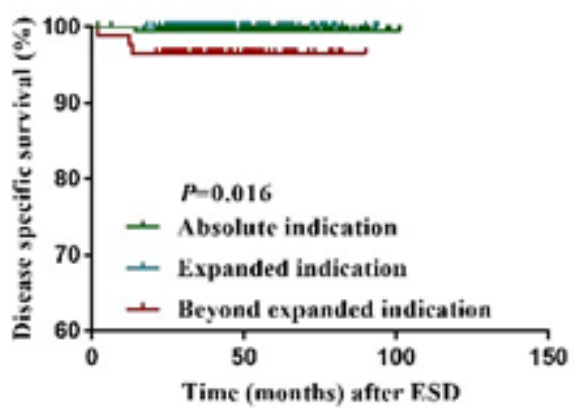

$\mathrm{D}$
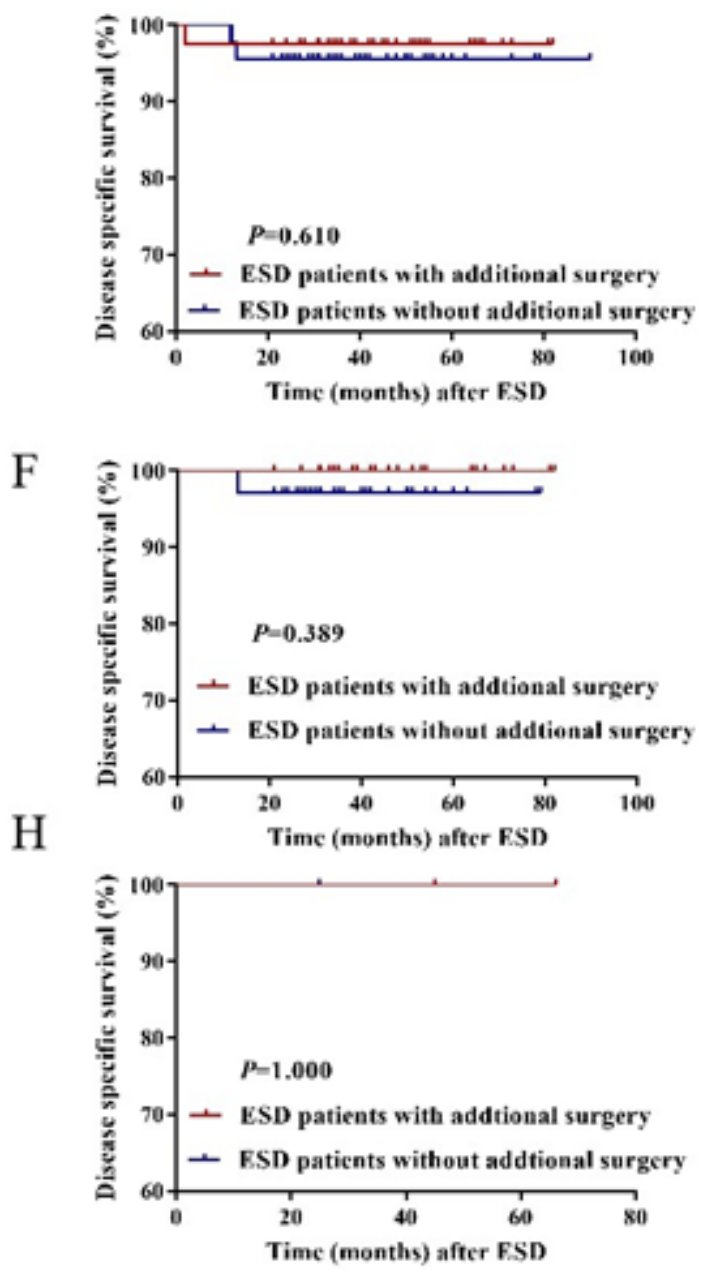

\section{Figure 1}

Kaplan-Meier plots of survival among patients after ESD. A: Overall survival between three groups. B: Disease specific survival between three groups. C: Overall survival between BEI patients with or without additional surgery. D: Disease specific survival between BEl patients with or without additional surgery. E. The eCura system score of the BEl patients with or without additional surgery. F. The disease specific survival of the BEl patients with or without additional surgery in low risk category. G. The disease specific survival of the BEl patients with or without additional surgery in intermediate risk category. $\mathrm{H}$. The disease specific survival of the BEl patients with or without additional surgery in high risk category. 


\section{Supplementary Files}

This is a list of supplementary files associated with this preprint. Click to download.

- Supplementary.docx

- Tables.docx 